\title{
LASER FREQUENCY COUNTING AND FREQUENCY MODULATION WIDTH MEASUREMENT FROM A FFT SIGNAL
}

\section{Petr Křen}

Czech Metrology Institute, V Botanice 4, CZ15072 Prague 5, Czech Republic( $\square$ pkren@cmi.cz, +420 257288 388)

\begin{abstract}
A new method of optical frequency beat counting based on fast Fourier transform (FFT) analysis is described. Signals with a worse signal-to-noise ratio can be counted correctly comparing to the conventional counting method of detecting each period separately. The systematic error of FFT counting below $10 \mathrm{~Hz}$ is demonstrated and can be decreased. Additionally the modulation width of a frequency-stabilized laser with high frequency modulation index can be simultaneously measured during a carrier frequency measurement against an optical frequency synthesizer or other laser.
\end{abstract}

Keywords: laser, modulation width, frequency measurement, optical frequency synthesizer.

(C) 2012 Polish Academy of Sciences. All rights reserved

\section{Introduction}

The optical beat which was first observed by A. Javan [1] is a crucial measurement tool for optical frequency standards. The frequency difference (radiofrequency beat) between two continuous wave lasers is generated in a detector by means of non-linear response to the electric field of the laser radiation. A measurement of the beat frequency of a frequencystabilized laser with a single component of femtosecond comb from an optical frequency synthesizer developed by T.W. Hänsch and J.L. Hall [2, 3] allows easy traceability of lasers for length measurements to the SI second.

A new fast Fourier transform (FFT) method for counting frequency of this beat (or offset frequency beat) is described in the article below. The main advantage of this method compared to the conventional counting with wide frequency bandwidth is that it is capable of correct counting weaker (frequency modulated or non-modulated) beat signals (down to $5 \mathrm{~dB}$ at $333 \mathrm{kHz}$ bandwidth which is about $10 \mathrm{~dB}$ better than the limit of conventional counting). This property can be realized because the noise of optical frequencies $h$ (less than the modulation width or frequency instability of the beat). In other wordsobserved in the beat is relatively reduced for measurement with a narrower frequency bandwidt, counting a larger number of similar periods together allows the relative noise reduction by an averaging. The minimal signal-to-noise ratio of FFT method is also better than the published levels used for a tracking oscillator assisted counting $[4,5]$.

\section{Setup and principle}

The measurement setup is shown in Fig. 1. The laser beat frequency is generated in an avalanche photodiode and measured by a conventional counter in a standard setup. If the signal is not sufficient for correct counting, a tracking oscillator is used by other laboratories to improve the conventional counter performance (dotted line). However, the conventional counter and tracking oscillator can be replaced by an analog-to-digital converter with FFT 
counting (dashed line). An analog-to-digital converter (Handyscope HS3) with $100 \mathrm{MHz}$ sampling (8-bit resolution) was used in this experiment. The measurement with this simple converter contains a dead time between individual data evaluations. Thus the noise of measurement is worse than that of the conventional stream measurements. The dead time outweighs the measurement time by the ratio of about 100:1 in this setup due to the time necessary for calculations. Thus in the case of FFT counting, all $1 \mathrm{~s}$ samples (or longer) will in fact stand for shorter samples in the article below. The frequency accuracy of the converter can be assured by locking the analog-to-digital converter clock frequency or by simultaneously counting the reference frequency (e.g. $10 \mathrm{MHz}$ ) in the second channel of the converter device.

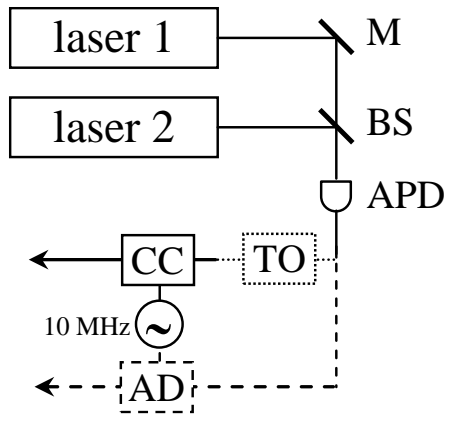

Fig. 1. The measurement setup ( $\mathrm{M}$ - mirror, BS - beam splitter, APD - avalanche photodiode, TO - tracking oscillator, $\mathrm{CC}$ - conventional counter, $\mathrm{AD}$ - analog-to-digital converter, $10 \mathrm{MHz}$ - reference frequency).

The laser radiofrequency $(\mathrm{RF})$ beat measurements were performed on an optical frequency comb (MenloSystems FC8004) with a GPS-disciplined Rb frequency standard [6]. The frequency is ordinarily measured with the dead-time-free counters of the fs-comb system. This RF beat between fs-comb and measured laser is displayed in the Fourier spectrum during measurement. The frequency of beat can be also adjusted into a selected frequency range by a slight change of repetition rate of the femtosecond laser. The desired frequency range for the FFT counting setup is from a few $\mathrm{MHz}$ to $50 \mathrm{MHz}$ (for the converter used here).

The RF beat signal for conventional counters is now also directly connected to the converter input. A single memory block of values obtained from the analog-to-digital converter contains voltage values of the beat signal. The length of this block can be arbitrary for non-modulated signals, but must be a length of a single period (or its multiple) of frequency modulation in case of frequency modulated lasers. (Their frequency modulation period is often known or can be easily measured. And then the FFT period selection gives an advantage over the tracking oscillator technique.) The measurement data obtained from the memory of the converter are further split into consequent FFT blocks. For each FFT block, the Blackman-Nuttall window function is applied for error suppression in the following determination of peak frequency. The length of this block must be properly chosen because the laser frequency change within the FFT block must be smaller than the bandwidth corresponding to the FFT block length. However, longer FFT blocks allow counting weaker signals due to the enhanced noise reduction. For a signal with frequency modulation of $1 \mathrm{kHz}$ we obtain for example $40 \mu$ s length of blocks for a width of $200 \mathrm{kHz}_{\mathrm{p}-\mathrm{p}}$ and $4 \mu \mathrm{s}$ length of blocks for a width up to $10 \mathrm{MHz}_{\mathrm{p}-\mathrm{p}}$. For a frequency modulation period of $120 \mu \mathrm{s}$ and width $6 \mathrm{MHz}_{\mathrm{p}-\mathrm{p}}$ we obtain a $2.5 \mu$ s FFT block length.

FFT counting of the optical beat frequency works if the difference in optical carrier frequency between the two lasers does not vary in the sub-millisecond range by more than the measurement bandwidth. This short-term stability is often associated with the mechanical 
properties of the optical cavity of each laser which prevent sudden changes in carrier frequency. Thus we can assume that the number of periods can be evaluated from the FFT peak frequency and phase within a short time interval (containing hundreds or thousands of periods of the beat signal). This radiofrequency peak must be evaluated by using amplitudes of adjacent frequency values to obtain better resolution than FFT bin width. As an interpolation we can use parabolic fitting. Nevertheless the systematic frequency shifts of the maximum can be substantial. Therefore the sixth order polynomial is used for (seven) amplitude values closest to the spectral maximum. The number of values for spectral lines broadened by windowing is selected to minimize the influence of FFT bins at line wings. Afterward the frequency position of the maximum is correctly calculated. The average peak frequency from all FFT blocks is used as carrier frequency result for a single period. Nevertheless the FFT peak frequency during the modulation period can be tracked. We can evaluate the modulation width from instantaneous frequency values of FFT peak positions during this modulation period (for example $1 \mathrm{~ms}$ ). The amplitude (modulation width) and phase of modulation are simply evaluated from the sine and the cosine Fourier coefficient values for a given modulation frequency as a function of time.

The advantage of this kind of counting is that the signal of each single period of the beat frequency may not be above the noise level. The weak signal observable on a spectrum analyzer with a given bandwidth can be counted correctly. Furthermore, the user can select an appropriate frequency range and avoid possible errors from a disturbing signal.

\section{Carrier frequency measurement}

The properties of FFT counting were tested with a signal from a RF generator (model HP 8647A) connected directly to the converter. The RF frequency with an amplitude of $+10 \mathrm{dBm}$, modulation frequency $1 \mathrm{kHz}$ and nominal modulation width $200 \mathrm{kHz}_{\mathrm{p}-\mathrm{p}}$ was used for this test. The average systematic error of measurement is below $100 \mathrm{~Hz}$ for carrier frequency and below $300 \mathrm{~Hz}$ for modulation width peak-to-peak. The source of this error is the finite measurement of frequency beat between the sampling rate with given FFT bandwidth and the measured carrier frequency with a given modulation width. The two standard deviations of noise for about $0.01 \mathrm{~s}$ samples (calculated within $1 \mathrm{~s}$ ) is about $20 \mathrm{~Hz}$ for a carrier frequency with $200 \mathrm{kHz}$-p modulation and about $6 \mathrm{~Hz}$ without applied modulation (residual modulation of the generator was slightly above $20 \mathrm{~Hz}_{\mathrm{p}-\mathrm{p}}$ ). These values are independent of the selection of the number of FFT blocks (in a reasonable range) and also they are not proportional to the carrier frequency value and its amplitude. The corresponding Allan standard deviations of carrier frequency measurements are shown in Fig. 2.

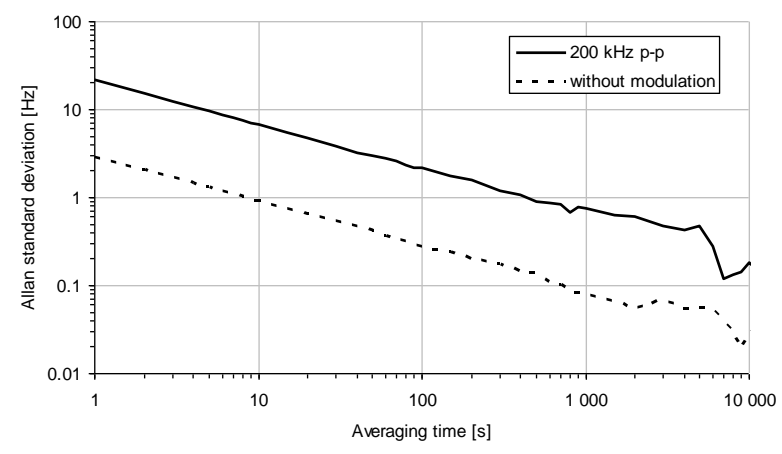

Fig. 2. The noise properties of carrier frequency counting (here for a carrier frequency of $10 \mathrm{MHz}$ ). 
The systematic error can be further suppressed by calculation of amplitudes for the demodulated carrier signal over a whole modulation period. The initial values of parameters such as carrier frequency $f_{C}$ and frequency modulation width amplitude $A_{M}$ and its phase $\varphi_{M}$ are taken from the previous FFT block analysis (for a known frequency of modulation $f_{M}$ ). The Fourier amplitude $A_{C}$ for the given modulated carrier frequency is then

$$
\begin{gathered}
A_{\mathrm{sin}}=\sum_{i=1}^{N} D_{i} W_{i} \sin \left(2 \pi f_{C} t_{i}+\frac{A_{M}}{f_{M}} \cos \left(2 \pi f_{M} t_{i}+\varphi_{M}\right)\right), \\
A_{\mathrm{cos}}=\sum_{i=1}^{N} D_{i} W_{i} \cos \left(2 \pi f_{C} t_{i}+\frac{A_{M}}{f_{M}} \cos \left(2 \pi f_{M} t_{i}+\varphi_{M}\right)\right), \\
A_{C}=2 \pi \sqrt{A_{\mathrm{sin}}^{2}+A_{\mathrm{cos}}^{2}} / N,
\end{gathered}
$$

where $D_{i}$ are digital data of the total number of $N$ obtained from the analog-to-digital converter at times $t_{i}$ and $W_{i}$ is the window function. These carrier frequency amplitudes can be calculated for slightly modified input parameters and the new corrected values will be determined for maximal amplitude of the carrier frequency. After the first iteration the systematic error of carrier frequency is below $10 \mathrm{~Hz}$. However the noise of parameters is not reduced. And the systematic error is also not decreased by the second iteration.

Nevertheless this method can be further improved for primary optical frequency standards by a fast (e.g. $100 \mathrm{MHz}$ sampling) stream FFT measurement currently available on the market. Using a high-end field-programmable gate array (FPGA) FFT analysis with special architecture the FFT counting can be exactly correct (without dead time needed for data transfer and calculations in a personal computer). The level of errors achieved above (i.e. less than a single converter sample) allow correct identification of phase and number of periods within a selected part of data stream and thus error free counting (i.e. without cycle slips).

If the streaming FFT measurement is not implemented, a further reduction of systematic errors of carrier frequency calculated from individual FFT blocks can be obtained in the following way. The parabolic fitting of maximum symmetry in frequency values is less affected by systematic errors. Nevertheless it is not possible to guarantee that the measured frequency will be exactly the frequency of a FFT bin (see Fig. 3). Thus the estimation $f_{e}$ of frequency position of the maximum from the FFT bin interpolation (with a bandwidth e.g. $100 \mathrm{kHz}$ ) can be used as the initial value for symmetric peak fitting. From a calculation of Fourier amplitudes for frequencies $f_{e}-\Delta, f_{e}$ and $f_{e}+\Delta$ (where the difference $\Delta$ is e.g. $1 \mathrm{kHz}$ ) we can obtain a new estimation $f_{e}$ with a systematic error down to $0.1 \mathrm{~Hz}$ (for an hour average of estimated carrier frequencies from millions independent data blocks). This small error value was obtained in several frequency ratio tests of different RF generator frequency outputs and its $10 \mathrm{MHz}$ reference frequency. Although this method does not change (improve) the Allan variance, it sufficiently reduces the uncertainty.

Afterwards the FFT counting was tested on measurements of a real noisy signal - RF beat of iodine frequency stabilized He-Ne laser (Winters Model 100) with fs-comb. The frequency of this beat was set close to $30 \mathrm{MHz}$ and its amplitude was about $-10 \mathrm{dBm}$. The signal-tonoise ratio for this measurement was about $20 \mathrm{~dB}$ for a $333 \mathrm{kHz}$ bandwidth. The measurement results from both methods are shown in Fig. 4. 


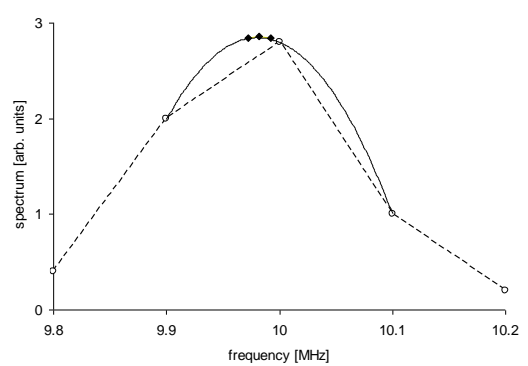

Fig. 3. An illustration of the difference between FFT (hollow points) and FT amplitudes symmetrically to the frequency center (filled points).

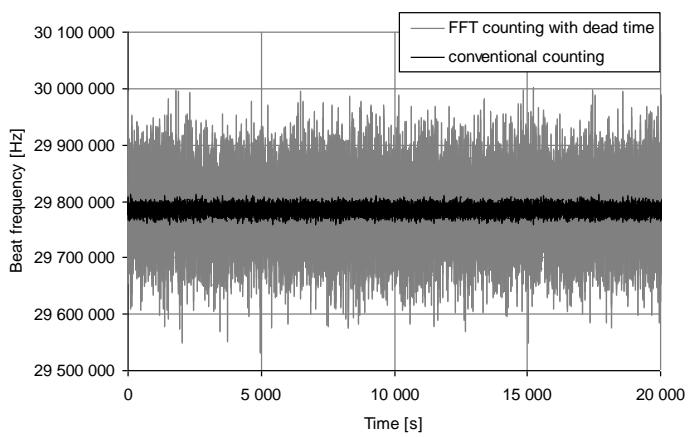

a)

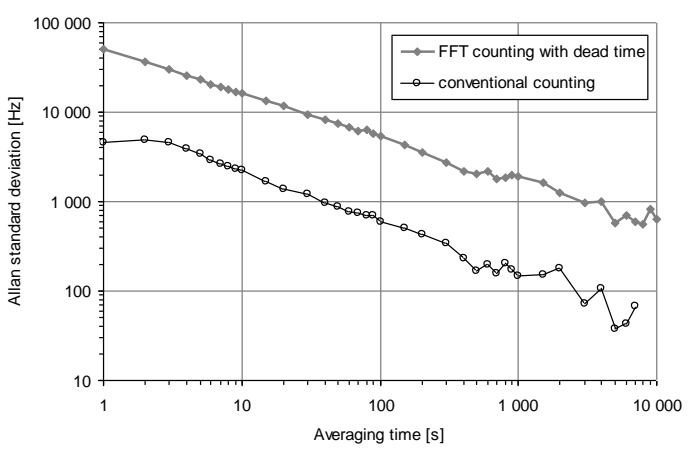

b)

Fig. 4. a) Example of frequency counting of the beat between a iodine frequency stabilized He-Ne laser and fscomb. b) Corresponding Allan standard deviation.

The measured instability by current implementation of the FFT method is worse than the intrinsic instability of the beat signal due to dead time for calculations. The $1 \mathrm{~s}$ sample of FFT counting in charts corresponds to about $10 \mathrm{~ms}$ of processed sample length (i.e. the standard deviation is 10 times worse for the square-root averaging of white frequency noise).

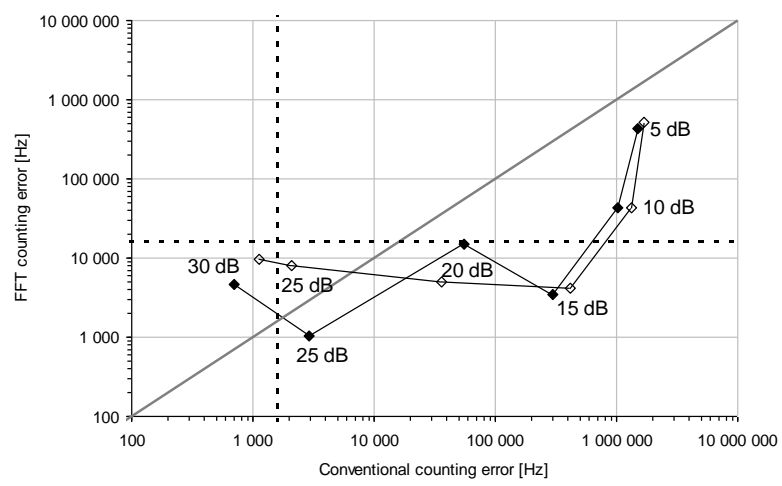

Fig. 5. Error comparison of both counting methods (for different FFT bandwidths: $333 \mathrm{kHz}$ filled points and 83 $\mathrm{kHz}$ open points). Each point corresponds to a decrease of signal-to-noise ratio (from about $30 \mathrm{~dB}$ at $20 \mathrm{kHz}$ bandwidth) of about $5 \mathrm{~dB}$ (from left to right). The dashed lines represent the statistic error of 1 minute measurements for each counting method.

While the signal-to-noise ratio of about $15 \mathrm{~dB}$ (at $333 \mathrm{kHz}$ bandwidth) is the limit for correct conventional counting, this FFT method allows counting down to the ratio of $5 \mathrm{~dB}$. I.e. the FFT method counting is still correct for about $10 \mathrm{~dB}$ weaker signals than conventional counting. Results of a correct counting capability test are shown in Fig. 5. Each point 
represents simultaneous FFT and conventional counting measurement of 1 minute length. The decrease of signal-to-noise ratio for the beat signal results in a worse counting. We can see at the fourth point from the left that the error of FFT counting is still correct within the statistic error of $16 \mathrm{kHz}(2 \sigma)$ whereas the error of conventional counting is more than $200 \mathrm{kHz}$ from the expected value obtained from previous correct counting results for this laser.

And finally a long-term test of counting was carried out. The error of frequency measurement of a He-Ne laser by this method compared to dead-time-free counting (with sufficient signal level) is less than $1 \mathrm{kHz}$ for a few hours averaging. This value is within the statistical error of this measurement and thus the FFT method was validated for ordinary measurement to this level.

\section{Modulation width measurement}

An additional advantage of this method is the capability of modulation width measurement. Frequency stabilized lasers measured by a fs-comb are often frequencymodulated. The modulation parameters are described in the Recommendation of CIPM-CCL [7] because the absolute frequency of a stabilized laser is sensitive to the width of modulation. Moreover this method allows measuring the modulation width of the beat signal in real time and tracking the corresponding frequency shift for each measurement.

The most common laser for precise length measurements is a He-Ne $633 \mathrm{~nm}$ frequencystabilized laser. The laser used for the modulation width measurements has a modulation frequency of $8.33 \mathrm{kHz}\left(120 \mu\right.$ s period) with nominal modulation width of $6 \mathrm{MHz}_{\mathrm{p}-\mathrm{p}}$ from the Recommendation. The deviation from the nominal value was measured to be less than $0.1 \%$ with long term drift below $0.02 \%$ per hour (see Fig. 6). The standard deviation of modulation width measured on different iodine components (measured within a few hours) was below $1 \mathrm{kHz}$. Moreover, it was previously tested that the difference of modulation width measurements is always less than $1 \%$ compared to the off-line method published in [8]. For a modulated signal with a high frequency modulation index (much more greater than one) the uncertainty of the FFT method described here is also better compared to values obtained by methods from commercial spectrum analyzers. The digital demodulation of FFT maxima during a selected modulation period does not suffer from systematic errors such as nonzero linewidth and bandwidth. Another advantage of the FFT method is the feature that the measurement does not need the second continuous wave laser of the same type (wavelength) to measure the modulation width as is needed in the above-mentioned off-line method.

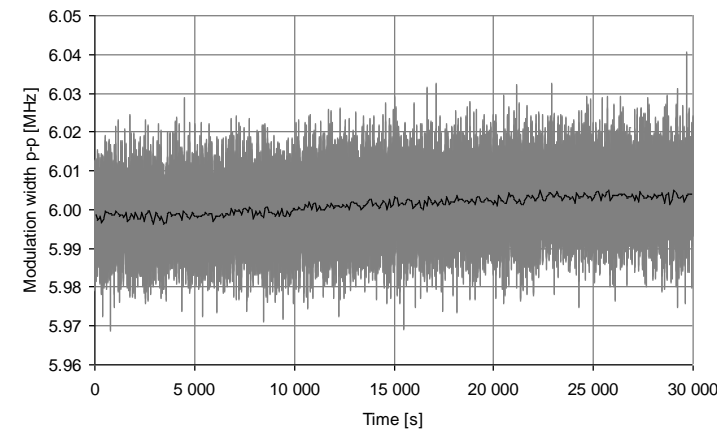

a)

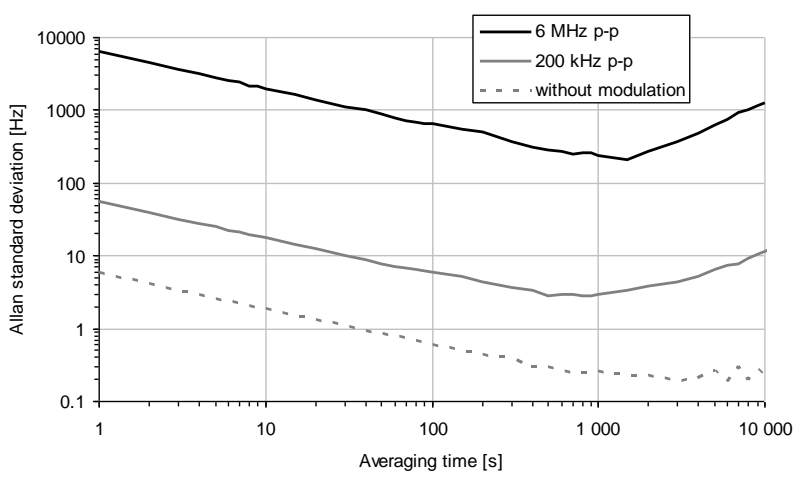

b)

Fig. 6. a) Modulation width of a He-Ne laser as a function of time. (1 s and $100 \mathrm{~s}$ sample length with about $99 \%$ of dead time) b) Corresponding Allan standard deviation (black line) compared to the measured variations for the signal from a RF generator (in grey) with modulation $200 \mathrm{kHz}_{\mathrm{p}-\mathrm{p}}$ (solid line) or almost non-modulated (dashed line). 
When we apply demodulation based on values from FFTs we can obtain instantaneous values for the carrier frequency (shown in Fig. 7a). We can see that the frequency changes between FFT blocks are smaller than the value of a correctly selected FFT bandwidth (here for example $666 \mathrm{kHz}$ ). This smooth change of carrier frequency thus confirms the FFT counting assumption from section 2. We can also observe that the Allan standard deviation of beat frequency between a He-Ne laser and RF oscillator disciplined fs-comb is flat (flicker frequency noise) in this range. However the carrier frequency of some He-Ne lasers can be disturbed. The laser optical frequency is modulated by a switching frequency (here about $25 \mathrm{kHz}$ ) of the high-voltage power supply of the laser tube (with modulation width up to $100 \mathrm{kHz}$ ). Nevertheless the Allan standard deviation of beat between the two He-Ne lasers (model Renishaw XL-80) without internal iodine cell exhibits values about $1 \mathrm{kHz}$ in the submillisecond range if the unwanted frequency modulation is subtracted (see Fig. 7b).

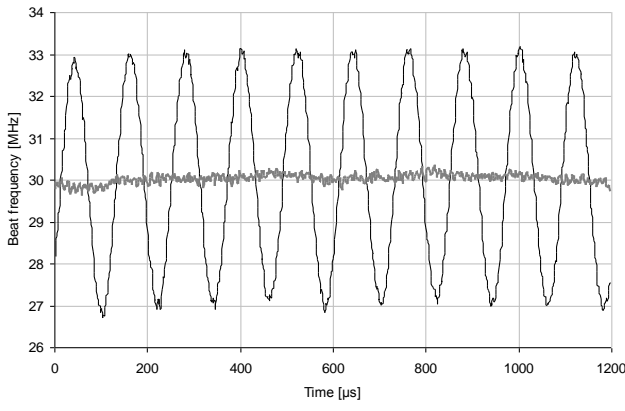

a)

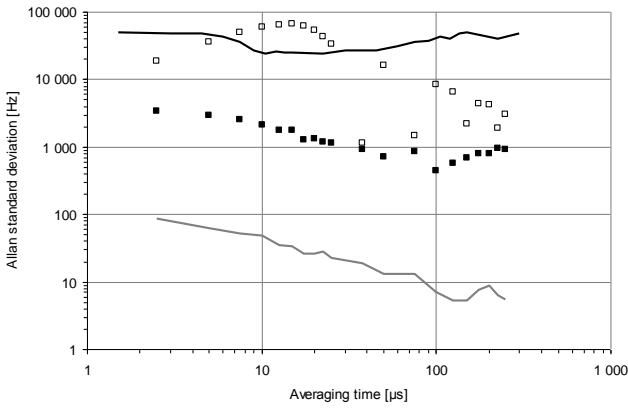

b)

Fig. 7. a) Black line shows frequency modulation of the beat between fs-comb and He-Ne laser and the grey line shows residuals after subtraction of modulation (i.e. stability of carrier frequency). b) Corresponding Allan standard deviation (black line) of carrier frequency (for a single $1.2 \mathrm{~ms}$ interval) compared to FFT counting capability for $10 \mathrm{MHz}$ signal of reference frequency (grey line). The Allan standard deviation for a beat frequency between two He-Ne lasers is plotted for comparison - with unwanted frequency modulation (open squares) and after its subtraction (filled squares).

\section{Conclusions}

A common counter with conventional counting is often limited by noise which is disturbing the trigger level and its hysteresis. The signal-to-noise ratio can be improved by filtering. However analog filters are not so versatile and convenient for counting the frequency of modulated signals. A digital filter is a simple and effective solution. And together with FFT, a weaker signal can be counted due to digital decrease of the counter frequency bandwidth.

The new useful tool for optical frequency beat measurement was successfully introduced. Results of the first simple realization are sufficient for secondary optical frequency standards and can improve their repeatability. Beat signals weaker by $10 \mathrm{~dB}$ (or more) can be now measured without loss of calibration accuracy compared to conventional counters. The modulation width of lasers can be measured simultaneously with their frequency deviation from the frequency standard and thus corrected for their influence on the carrier frequency and can improve their repeatability. This FFT counting is practical for frequency filtering capability and further simplifies measurements with optical frequency synthesizers.

\section{References}

[1] Javan, A., Ballik, E.A., Bond, W.L. (1962). Frequency characteristics of a continuous-wave He-Ne optical maser. J. Opt. Soc. Am., 52, 96-98. 
[2] Reichert, J., Niering, M., Holzwarth, R., Weitz, M., Udem, Th., Hänsch, T. W. (2000). Phase coherent vacuum-ultraviolet to radio frequency comparison with a mode-locked laser. Phys. Rev. Lett., 84, $3232-3235$.

[3] Jones, D.J., Diddams, S.A., Ranka, J.K., Stentz, A., Windeler, R.S., Hall, J.L., Cundiff, S.T. (2000). Carrier-envelope phase control of femtosecond mode-locked lasers and direct optical frequency synthesis. Science, 288, 635-639.

[4] Ye, J., Yoon, T.H., Hall, J.L., Madej, A.A., Bernard, J.E., Siemsen, K.J., Marmet, L., Chartier, J.M., Chartier, A. (2000). Accuracy comparison of absolute optical frequency measurement between harmonicgeneration synthesis and a frequency-division femtosecond comb. Phys. Rev. Lett., 85, 3797-3800.

[5] Stenger, J., Binnewies, T., Wilpers, G., Riehle, F., Telle, H.R., Ranka, J.K., Windeler, R.S., Stentz, A.J. (2001). Phase-coherent frequency measurement of the $\mathrm{Ca}$ intercombination line at $657 \mathrm{~nm}$ with a Kerrlens mode-locked femtosecond laser. Phys. Rev. A, 63 021802(R).

[6] Balling, P., Křen, P. (2008). Absolute frequency measurements of wavelength standards $532 \mathrm{~nm}, 543 \mathrm{~nm}$, $633 \mathrm{~nm}$ and $1540 \mathrm{~nm}$. EPJ D, 48, 3-10.

[7] Felder, R. (2005). Practical realization of the definition of the metre, including recommended radiations of other optical frequency standards (2003). Metrologia, 42, 323, http://www.bipm.org/en/ committees/cc/ccl/mep.html.

[8] Balling, P. (2001). Measurement of frequency modulation amplitude of lasers to 1 part in $10^{3}$. Metrologia, 38, 297-299. 